\title{
ON CERTAIN PRODUCTS OF PERMUTABLE SUBGROUPS
}

\author{
A. BALLESTER-BOLINCHES $\mathbb{D}^{\otimes}$, S. Y. MADANHA ${ }^{\mathbb{D}}$, T. M. MUDZIIRI SHUMBA $\mathbb{}$ \\ and M. C. PEDRAZA-AGUILERA (i)
}

(Received 28 April 2021; accepted 8 July 2021; first published online 16 August 2021)

Dedicated to the memory of Alexander Grant Robertson Stewart

\begin{abstract}
In this paper, we study the structure of finite groups $G=A B$ which are a weakly mutually $s n$-permutable product of the subgroups $A$ and $B$, that is, $A$ permutes with every subnormal subgroup of $B$ containing $A \cap B$ and $B$ permutes with every subnormal subgroup of $A$ containing $A \cap B$. We obtain generalisations of known results on mutually $s n$-permutable products.
\end{abstract}

2020 Mathematics subject classification: primary 20D10; secondary 20D20.

Keywords and phrases: mutually $s n$-permutable subgroups, supersoluble groups, widely supersoluble groups.

\section{Introduction}

All groups considered here will be finite.

Mutually permutable products, that is, products $G=A B$ such that $A$ permutes with every subgroup of $B$ and $B$ permutes with every subgroup of $A$, have been extensively studied by many authors [3]. In recent years, some other permutability connections between the factors have also been considered. In particular, the rich normal structure of a mutually permutable product of two nilpotent groups [3, Ch. 5] has motivated interest in the study of mutually $s n$-permutable products.

DEFINITION 1.1. We say that a group $G=A B$ is the mutually $s n$-permutable product of the subgroups $A$ and $B$ if $A$ permutes with every subnormal subgroup of $B$ and $B$ permutes with every subnormal subgroup of $A$.

Carocca [5] showed that a mutually sn-permutable product of two soluble groups is also soluble. In [1], the authors analyse the structure of mutually sn-permutable products and prove the following extension of a classical result of Asaad and Shaalan [2].

(C) The Author(s), 2021. Published by Cambridge University Press on behalf of Australian Mathematical Publishing Association Inc. This is an Open Access article, distributed under the terms of the Creative Commons Attribution licence (https://creativecommons.org/licenses/by/4.0/), which permits unrestricted re-use, distribution, and reproduction in any medium, provided the original work is properly cited. 
THEOREM 1.2 [1, Theorem B]. Let $G=A B$ be the mutually sn-permutable product of the subgroups $A$ and $B$, where $A$ is supersoluble and $B$ is nilpotent. If $B$ permutes with each Sylow subgroup of $A$, then the group $G$ is supersoluble.

Following [8], we say that a subgroup $H$ of a group $G$ is $\mathbb{P}$-subnormal in $G$ whenever either $H=G$ or there exists a chain of subgroups $H=H_{0} \leq H_{1} \leq \cdots \leq H_{n-1} \leq H_{n}=$ $G$ such that $\left|H_{i}: H_{i-1}\right|$ is a prime for every $i=1, \ldots, n$. It turns out that supersoluble groups are exactly those groups in which every subgroup is $\mathbb{P}$-subnormal. Having in mind this result and the influence of the embedding of Sylow subgroups on the structure of a group, the following extension of the class of supersoluble groups introduced in [8] seems to be natural.

Definition 1.3. A group $G$ is called widely supersoluble, $w$-supersoluble for short, if every Sylow subgroup of $G$ is $\mathbb{P}$-subnormal in $G$.

The class of all finite $w$-supersoluble groups, denoted by $w \mathcal{U}$, is a saturated formation of soluble groups containing $\mathcal{U}$, the class of all supersoluble groups, which is locally defined by a formation function $f$, such that for every prime $p, f(p)$ is composed of all soluble groups $G$ whose Sylow subgroups are abelian of exponent dividing $p-1$ [8, Theorems 2.3 and 2.7]. Not every group in $w \mathcal{U}$ is supersoluble [8, Example 1]. However, every group in $w \mathcal{U}$ has an ordered Sylow tower of supersoluble type [8, Proposition 2.8].

In [4], mutually sn-permutable products in which the factors are $w$-supersoluble are analysed. The following extension of Theorem 1.2 holds.

THEOREM 1.4 [4, Theorem 4]. Let $G=A B$ be the mutually sn-permutable product of the subgroups $A$ and $B$, where $A$ is $w$-supersoluble and $B$ is nilpotent. If $B$ permutes with each Sylow subgroup of $A$, then the group $G$ is w-supersoluble.

Assume that $G=A B$ is the mutually sn-permutable product of the subgroups $A$ and $B$. Then, by [3, Proposition 4.1.16 and Corollary 4.1.17], $A \cap B$ is subnormal in $G$ and permutes with every subnormal subgroup of $A$ and $B$. Assume now that $G=A B$ and $A \cap B$ satisfy this condition. Then $G$ is the mutually $s n$-permutable product of $A$ and $B$ if and only if $A$ permutes with every subnormal subgroup $V$ of $B$ such that $A \cap B \leqslant V$ and $B$ permutes with every subnormal subgroup $U$ of $A$ such that $A \cap B \leqslant U$. This motivates the following definition.

Definition 1.5. Let $A$ and $B$ be two subgroups of a group $G$ such that $G=A B$. We say that $G$ is the weakly mutually $s n$-permutable product of $A$ and $B$ if $A$ permutes with every subnormal subgroup $V$ of $B$ such that $A \cap B \leqslant V$ and $B$ permutes with every subnormal subgroup $U$ of $A$ such that $A \cap B \leqslant U$.

Obviously, mutually sn-permutable products are weakly mutually $s n$-permutable, but the converse is not true in general, as the following example shows.

EXAMPLE 1.6. Let $G=\Sigma_{4}$ be the symmetric group of degree 4. Consider a maximal subgroup $A$ of $G$ which is isomorphic to $\Sigma_{3}$, and $B=A_{4}$, the alternating group of 
degree 4 . Then $G=A B$ is the weakly mutually $s n$-permutable product of the subgroups $A$ and $B$. However, the product is not mutually $s n$-permutable because $A$ does not permute with a subnormal subgroup of order 2 of $B$.

The first goal of this paper to prove weakly mutually sn-permutable versions of the aforesaid theorems. We show that Theorem 1.4 holds for weakly mutually sn-permutable products.

THEOREM A. Let $G=A B$ be the weakly mutually sn-permutable product of the subgroups $A$ and $B$, where $A$ is $w$-supersoluble and $B$ is nilpotent. If $B$ permutes with each Sylow subgroup of $A$, then the group $G$ is w-supersoluble.

The next corollary follows from the proof of Theorem A and generalises Theorem 1.2.

COROLlARY B. Let $G=A B$ be the weakly mutually sn-permutable product of the subgroups $A$ and $B$, where $A$ is supersoluble and $B$ is nilpotent. If $B$ permutes with each Sylow subgroup of $A$, then the group $G$ is supersoluble.

The second part of the paper is concerned with weakly mutually sn-permutable products with nilpotent derived subgroups. Our starting point is the following extension of a classical result of Asaad and Shaalan [2].

THEOREM 1.7 [1, Theorem C]. Let $G=A B$ be the mutually sn-permutable product of the supersoluble subgroups $A$ and $B$. If the derived subgroup $G^{\prime}$ of $G$ is nilpotent, then $G$ is supersoluble.

A natural question is whether this result is true for weakly mutually sn-permutable products under the same conditions. The following example answers this question negatively.

EXAMPLE 1.8. Let $G=\left\langle a, b, c: a^{5}=b^{5}=c^{6}=1, a b=b a, a^{c}=a^{2} b^{3}, b^{c}=a^{-1} b^{-1}\right\rangle \simeq$ $\left[C_{5} \times C_{5}\right] C_{6}$. Then $G=A B$ is the weakly mutually sn-permutable product of $A=\langle c\rangle$ and $B=[\langle a\rangle \times\langle b\rangle]\left\langle c^{3}\right\rangle$. Note that $B$ is a normal subgroup of $G$; therefore, it permutes with every subgroup of $A$. Moreover, $A \cap B=\left\langle c^{3}\right\rangle$ and the unique subnormal subgroup of $B$ containing $A \cap B$ is the whole of $B$. It is not difficult to see that $B$ is supersoluble. Therefore, $A$ and $B$ are supersoluble and $G^{\prime}$ is nilpotent. Moreover, $A$ is nilpotent and $B$ is a normal subgroup of $G$. Thus, in particular, it permutes with every Sylow subgroup of $A$.

However, an additional assumption allows us to get supersolubility.

THEOREM C. Let $G=A B$ be the weakly mutually sn-permutable product of the supersoluble subgroups $A$ and $B$. If B permutes with each Sylow subgroup of $A$, A permutes with every Sylow subgroup of $B$ and the derived subgroup $G^{\prime}$ of $G$ is nilpotent, then $G$ is supersoluble. 
By [7, Theorem 2.6], a group $G$ is $w$-supersoluble if and only if every metanilpotent subgroup of $G$ is supersoluble. In particular, if we have a group with $G^{\prime}$ nilpotent, every $w$-supersoluble subgroup is supersoluble. Therefore, the following result is clear.

COROLlary D. Let $G=A B$ be the weakly mutually sn-permutable product of the w-supersoluble subgroups $A$ and $B$. If $B$ permutes with each Sylow subgroup of $A$, $A$ permutes with every Sylow subgroup of $B$ and the derived subgroup $G^{\prime}$ of $G$ is nilpotent, then $G$ is w-supersoluble.

\section{Preliminary results}

In this section we will prove some results needed for the proofs of our main results. We start by showing that factor groups of weakly mutually sn-permutable products are also weakly mutually sn-permutable products.

LEMMA 2.1. Let $G=A B$ be the weakly mutually sn-permutable product of $A$ and $B$, and let $N$ be a normal subgroup of $G$. Then $G / N=(A N / N)(B N / N)$ is the weakly mutually sn-permutable product of $A N / N$ and $B N / N$.

Proof. Let us consider $G / N=(A N / N)(B N / N)$. Suppose that $H N / N$ is a subnormal subgroup of $A N / N$ such that $A N / N \cap B N / N \leqslant H N / N$. Note that $U=H N \cap A$ is a subnormal subgroup of $A$ such that $U N=H N$ and $A \cap B \leq U$. Since $U$ permutes with $B$, it follows that $H N=U N$ permutes with $B N$.

Interchanging $A$ and $B$ and arguing in the same manner proves the result.

LEMMA 2.2. Let $G=A B$ be the weakly mutually sn-permutable product of $A$ and $B$.

(a) If $H$ is a subnormal subgroup of $A$ such that $A \cap B \leqslant H$, then $H B$ is a weakly mutually sn-permutable product of $H$ and $B$.

(b) If $A \cap B=1$, then $G=A B$ is a totally sn-permutable product of $A$ and $B$.

PROOF. Since every subnormal subgroup of $H$ is a subnormal subgroup of $A$, it follows that $B$ permutes with every subnormal subgroup $L$ of $H$ such that $A \cap B \leqslant L$. On the other hand, let $M$ be a subnormal subgroup of $B$ such that $A \cap B \leqslant M$. Then we have $H M=H(A \cap B) M=(A \cap H B) M=A M \cap H B=M A \cap B H=M(A \cap B H)=$ $M(A \cap B) H=M H$. Hence $H B$ is a weakly mutually sn-permutable product of $H$ and $B$.

For (b), every subnormal subgroup of $A$ permutes with $B$ by (a) and every subnormal subgroup of $B$ permutes with $A$. So $G=A B$ is the mutually sn-permutable product of $A$ and $B$. Hence $G=A B$ is the totally sn-permutable product of $A$ and $B$ since $A \cap B=1$.

Observe that Lemma 2.2 implies that if $G=A B$ is the weakly mutually $s n$-permutable product of $A$ and $B, H$ is a subnormal subgroup of $A$ such that $A \cap B \leqslant H$ and $K$ is a subnormal subgroup of $B$ such that $A \cap B \leqslant K$, then $H K$ is a weakly mutually sn-permutable product of $H$ and $K$. In the next result we analyse the 
behaviour of minimal normal subgroups of weakly mutually sn-permutable products containing the intersection of the factors.

LEMMA 2.3. Let $G=A B$ be the weakly mutually sn-permutable product of $A$ and $B$. If $N$ is a minimal normal subgroup of $G$ such that $A \cap B \leqslant N$, then either $A \cap N=$ $B \cap N=1$ or $N=(N \cap A)(N \cap B)$.

Proof. Observe that $A \cap N$ is a normal subgroup of $A$ such that $A \cap B \leqslant A \cap N$ and consequently $H=(A \cap N) B$ is a subgroup of $G$. Note that $N \cap H=N \cap(A \cap N) B=$ $(A \cap N)(B \cap N)$. Since $N \cap H$ is a normal subgroup of $H$, it follows that $B$ normalises $N \cap H=(A \cap N)(B \cap N)$.

By the same argument, $K=A(B \cap N)$ is a subgroup of $G$ such that $K \cap N=$ $A(B \cap N) \cap N=(A \cap N)(B \cap N)$. Moreover, $A$ normalises $K \cap N=(A \cap N)(B \cap N)$. It follows that $(A \cap N)(B \cap N)$ is a normal subgroup of $G$. By the minimality of $N$, $A \cap N=B \cap N=1$ or $N=(N \cap A)(N \cap B)$ as required.

LEMMA 2.4. Let $G=A B$ be the weakly mutually sn-permutable product of the subgroups $A$ and $B$, where $B$ is nilpotent. If $B$ permutes with each Sylow subgroup of $A$, then $A \cap B$ is a nilpotent subnormal subgroup of $G$.

Proof. It is clear that $A \cap B$ is nilpotent. The Sylow subgroups of $B$ are normal in $B$, so $A \cap B$ permutes with every Sylow subgroup of $B$. Let $A_{q}$ be a Sylow subgroup of $A$, with $q$ a prime dividing $|A|$. Since $B$ permutes with every Sylow subgroup of $A$, it follows that $B A_{q}$ is a subgroup of $G$. Hence $B A_{q} \cap A=A_{q}(A \cap B)$. Therefore $A \cap B$ permutes with every Sylow subgroup of $A$. Applying [3, Theorem 1.2.14(3)], $A \cap B$ is a subnormal subgroup of both $A$ and $B$. By [3, Theorem 1.1.7], $A \cap B$ is a subnormal subgroup of $G$.

LEMMA 2.5. Let $G=A B$ be the weakly mutually sn-permutable product of the subgroups $A$ and $B$, where $A$ is soluble and $B$ is nilpotent. If $B$ permutes with each Sylow subgroup of $A$, then the group $G$ is soluble.

ProOf. Suppose that the theorem is false, and let $G$ be a minimal counterexample. If $N$ is a minimal normal subgroup of $G$, then $G / N=(A N / N)(B N / N)$ is the weakly mutually $s n$-permutable product of the subgroups $A N / N$ and $B N / N$ by Lemma 2.1. Since $B N / N$ permutes with each Sylow subgroup of $A N / N$, it follows that $G / N$ is soluble by the minimality of $G$. Let $N_{1}$ and $N_{2}$ be two minimal subgroups of $G$. Then $G \cong G /\left(N_{1} \cap N_{2}\right)$ is soluble, a contradiction. Hence $G$ has a unique minimal normal subgroup $N$ of $G$ and we may assume that $N$ is nonabelian. This means that $\mathbf{F}(G)=1$.

On the other hand, $A \cap B \leqslant \mathbf{F}(G)$ using Lemma 2.4. Therefore $A \cap B=1$ and then $G=A B$ is the totally $s n$-permutable product of $A$ and $B$. The result then follows by applying [5, Theorem 6].

LEMMA 2.6 [1, Lemma 3]. Let $G$ be a primitive group and let $N$ be its unique minimal normal subgroup. Assume that $G / N$ is supersoluble. If $N$ is a p-group, where $p$ is the largest prime dividing $|G|$, then $N=\mathbf{F}(G)=\mathbf{O}_{p}(G)$ is a Sylow p-subgroup of $G$. 


\section{Main results}

We are ready to prove our main results.

Proof of Theorem A. Suppose the theorem is not true and let $G$ be a minimal counterexample. We shall prove our theorem in five steps.

(a) $G$ is a primitive soluble group with a unique minimal normal subgroup $N$ and $N=\mathbf{C}_{G}(N)=\mathbf{F}(G)=\mathbf{O}_{p}(G)$ for a prime $p$. Let $N$ be a minimal normal subgroup of $G$. By Lemma $2.1, G / N=(A N / N)(B N / N)$ is a weakly mutually sn-permutable product of $A N / N$ and $B N / N$ and it is clear that $B N / N$ permutes with every Sylow subgroup of $A N / N$. Moreover, $A N / N$ is $w$-supersoluble and $B N / N$ is nilpotent. By the minimality of $G$, it follows that $G / N$ is $w$-supersoluble. Note that $w \mathcal{U}$, the class of finite $w$-supersoluble groups, is a saturated formation of soluble groups by [8, Theorems 2.3 and 2.7]. This implies that $G$ is a primitive soluble group and so $G$ has a unique minimal normal subgroup $N$ with $N=\mathbf{C}_{G}(N)=\mathbf{F}(G)=\mathbf{O}_{p}(G)$ for some prime $p$ as required.

(b) The subgroup $B N$ is $w$-supersoluble and $1 \neq A \cap B \leqslant N$. If $A \cap B=1$, then the result follows by Lemma 2.2 and Theorem 1.4. Applying Lemma 2.4, it follows that $A \cap B$ is a nilpotent subnormal subgroup of $G$. Therefore $1 \neq A \cap B \leqslant \mathbf{F}(G)=N$ and so $N=(N \cap A)(N \cap B)$ by Lemma 2.3. Hence $N B=(N \cap A)(N \cap B) B=(N \cap A) B$ is a weakly mutually sn-permutable product of $N \cap A$ and $B$. Also note that $B$ permutes with every Sylow subgroup of $N \cap A$ (there is only one Sylow subgroup of $N \cap A$, which is $N \cap A)$. If $N B<G$, then $N B$ is $w$-supersoluble by the choice of $G$. So we may assume that $G=N B$. In this case, consider a subgroup $N_{1} \leqslant A \cap B \leqslant N$. Note that $N_{1}$ is normal in $N$ since $N$ is abelian. Hence $N=N_{1}^{G}=N_{1}^{N B}=N_{1}^{B} \leqslant B$ and $G=B$, a contradiction. Hence the result follows.

(c) $N$ is the Sylow $p$-subgroup of $G$ and $p$ is the largest prime dividing $|G|$. Let $q$ be the largest prime dividing $|G|$ and suppose that $q \neq p$. Suppose first that $q$ divides $|B N|$. Since $B N$ has a Sylow tower of supersoluble type, $B N$ has a unique Sylow $q$-subgroup, say $(B N)_{q}$. This means that $(B N)_{q}$ centralises $N$. Thus $(B N)_{q}=1$, since $\mathbf{C}_{G}(N)=N$, a contradiction.

We may assume that $q$ divides $|A|$ but does not divide $|B N|$. Since $A$ has a Sylow tower of supersoluble type, $A$ has a unique Sylow $q$-subgroup, $A_{q}$ say. This means that $A_{q}$ is normalised by $N \cap A$. Consider $A_{q}(N \cap B)=A_{q}(A \cap B)(N \cap B)$, a weakly mutually permutable product of $A_{q}(A \cap B)$ and $N \cap B$ by Lemma 2.2. Also $N \cap B$ permutes with each Sylow subgroup of $A_{q}(A \cap B)$. Suppose that $A_{q}(N \cap B)<G$. Then $A_{q}(N \cap B)$ is $w$-supersoluble by the choice of $G$. It follows that $A_{q}(N \cap B)$ has a unique Sylow $q$-subgroup since it has a Sylow tower of supersoluble type. In other words, $A_{q}$ is normalised by $N \cap B$. Hence $A_{q}$ is normalised by $(N \cap A)(N \cap B)=N$. This means that $A_{q}$ centralises $N$, a contradiction. We may assume that $A_{q}(N \cap B)=G$. Then $N \cap B=B$ and so $B$ is an elementary abelian $p$-group. Moreover, $A=A_{q}(A \cap B)$. Since $A \cap B$ is a Sylow $p$-subgroup of $A$ which is subnormal in $A$, it is normal in $A$. Hence $A \cap B$ is normal in $G$ because $A \cap B$ is normal in the abelian group $B$. By the 
minimality of $N$, it follows that $N=A \cap B$, that is, $G=A_{q}(N \cap B)=A_{q}(A \cap B)=A$, a contradiction. Therefore $p$ is the largest prime dividing $|G|$.

We now prove that $N$ is the Sylow $p$-subgroup of $G$. Since $G$ is a primitive soluble group, $G=N M$, where $M$ is a maximal subgroup of $G$ and $N \cap M=1$. Then $M \cong G / N$ is $w$-supersoluble. By [6, Theorem A.15.6], $\mathbf{O}_{p}(M)=1$. If $p$ divides $|M|$, then since $M$ has a Sylow tower of supersoluble type, $\mathbf{O}_{p}(M) \neq 1$, a contradiction. Hence $p$ does not divide $|M|$ and therefore $N$ is the unique Sylow $p$-subgroup of $G$.

(d) $N$ is contained in $A$ and $N$ is not contained in $B$. Suppose that $B$ is a $p$-group. Then $G=A N$. Let $N_{1} \leqslant A \cap B$. Since $B$ is abelian, $N \leq N_{1}^{G}=N_{1}^{A N}=N_{1}^{A} \leqslant A$ and so $G=A N=A$, a contradiction. So we may assume that $B$ is not a $p$-group. If $N$ is contained in $B$, then since $B$ is nilpotent and $N=\mathbf{C}_{G}(N)$, it follows that $B$ is a $p$-group, a contradiction. Therefore $N$ is not contained in $B$. Hence $B$ has a nontrivial Hall $p^{\prime}$-subgroup, $B_{p^{\prime}}$, which is normal in $B$. Consequently, $A B_{p^{\prime}}=A(A \cap B) B_{p^{\prime}}$ is a subgroup of $G$. Then $1 \neq B_{p^{\prime}}^{G} \leqslant A B_{p^{\prime}}$ and so $N \leqslant A B_{p^{\prime}}$. Hence $N \leqslant A$ as required.

(e) Final contradiction. Let $A_{p^{\prime}}$ be a Hall $p^{\prime}$-subgroup of $A$. If $A_{p^{\prime}}=1$, then $G=$ $B N$ is $w$-supersoluble by (b), a contradiction. Hence $A_{p^{\prime}} \neq 1$. Since $B$ permutes with every Sylow subgroup of $A$, it follows that $A_{p^{\prime}} B$ is a subgroup of $G$. But $N$ is not contained in $B$, so $A_{p^{\prime}} B$ is a proper subgroup of $G$. Since $N A_{p^{\prime}} B=G$, it follows that $N \cap A_{p^{\prime}} B=N \cap B$ is normal in $G$. The minimality of $N$ implies that $N=N \cap B$ or $N \cap B=1$. If $N=N \cap B$, we get a contradiction with (d). Therefore $N \cap B=1$, and then $A \cap B \leqslant N \cap B=1$, contradicting (b).

Proof of Theorem C. Assume the result is not true and let $G$ be a minimal counterexample. It is clear that $G^{\prime} \neq 1, A$ and $B$ are proper subgroups of $G$, and $G$ is a primitive soluble group. Hence there exists a unique minimal normal subgroup $N$ of $G$, such that $N=F(G)=C_{G}(N)$. Moreover, $G^{\prime}=N$. We may assume that $A^{\prime} \neq 1$ and $B^{\prime} \neq 1$, otherwise $A$ or $B$ is nilpotent and the result follows from Corollary B. If $A \cap B=1$, then $G$ is the mutually sn-permutable product of $A$ and $B$. By [1, Theorem C], the group is supersoluble, a contradiction. Thus we may assume $A \cap B \neq 1$. Since $A$ permutes with every Sylow subgroup of $B$ and $B$ permutes with every Sylow subgroup of $A$, it follows that $A \cap B$ permutes with every Sylow subgroup of $A$ and every Sylow subgroup of $B$. Hence $A \cap B$ is subnormal in $A$ and it is a subnormal subgroup of $B$. Let $N_{1}$ denote a minimal normal subgroup of $A$ such that $N_{1} \leq A^{\prime}$. Since $A$ is supersoluble, it is clear that $\left|N_{1}\right|=p$. Note that $N_{1}(A \cap B)$ is a subnormal subgroup of $A$. Therefore $B N_{1}(A \cap B)=B N_{1}$ is a subgroup of $G$. Now $1 \neq N_{1}^{G}=N_{1}^{B} \leq B N_{1}$. Hence $N \leq B N_{1}$ and then $N=N_{1}(N \cap B)$. Consequently, either $N_{1} \leq N \cap B$ or $N_{1} \leq N \cap B$. Denote $T=B N$. If $N_{1} \leq N \cap B$, then $T=B$ is a supersoluble normal subgroup of $G$. Assume $N_{1} \cap(N \cap B)=1$. Then $N \cap B$ is a maximal subgroup of $N$ and so $T$ is the weakly mutually sn-permutable product of $B$ and $N$. Consequently, $T$ satisfies the hypotheses of the theorem. If $T$ is a proper subgroup of $G$, then $T=B N$ is supersoluble. Assume that $G=B N$. Then $B$ is a maximal subgroup of $G$ such that $B \cap N=1, B^{\prime} \leq N \cap B=1$ and $B$ is nilpotent. By Corollary B, $G$ is supersoluble, contrary to assumption. Hence either $B$ is a normal subgroup of $G$ or $B N$ is a supersoluble normal subgroup of $G$. 
Arguing in an analogous manner with $A$ shows that if $A N$ is a proper subgroup of $G$, then it is supersoluble. Consequently if $B N$ and $A N$ are both proper subgroups of $G$, then $G$ is the product of two supersoluble normal subgroups with $G^{\prime}$ nilpotent. Then $G$ is supersoluble, a contradiction. Therefore we may assume that $G=B N$ or $G=A N$. Suppose without loss of generality that $G=B N$. Then $N \cap B$ is a normal subgroup of $G$. If $N \cap B=N$, then $G=B$, a contradiction. Hence $N \cap B=1$. Now $B^{\prime} \leq N \cap B=1$ and $B$ is nilpotent, the final contradiction.

\section{References}

[1] M. J. Alejandre, A. Ballester-Bolinches, J. Cossey and M. C. Pedraza-Aguilera, 'On some permutable products of supersoluble groups', Rev. Mat. Iberoam. 20 (2004), 413-425.

[2] M. Asaad and A. Shaalan, 'On the supersolvability of finite groups', Arch. Math. (Basel) 53 (1989), 318-326.

[3] A. Ballester-Bolinches, R. Esteban-Romero and M. Asaad, Products of Finite Groups (Walter De Gruyter, Berlin-New York, 2010).

[4] A. Ballester-Bolinches, W. M. Fakieh and M. C. Pedraza-Aguilera, 'On products of generalised supersoluble finite groups', Mediterr. J. Math. 16 (2019), 46.

[5] A. Carocca, 'On factorized finite groups in which certain subgroups of the factors permute', Arch. Math. (Basel) 71 (1998), 257-261.

[6] K. Doerk and T. O. Hawkes, Finite Soluble Groups (Walter De Gruyter, Berlin-New York, 1992).

[7] V. S. Monakhov, 'Finite groups with abnormal and U-subnormal subgroups', Sib. Math. J. 57 (2016), 352-363.

[8] A. F. Valisev, T. I. Valiseva and V. N. Tyutyanov, 'On the finite groups of supersoluble type', Sib. Math. J. 51 (2010), 1004-1012.

A. BALLESTER-BOLINCHES, Departament de Matemàtiques, Universitat de València, Dr. Moliner 50, 46100 Burjassot, València, Spain e-mail: adolfo.ballester@uv.es

S. Y. MADANHA, Department of Mathematics and Applied Mathematics, University of Pretoria, Pretoria, 0002, South Africa e-mail: sesuai.madanha@up.ac.za

T. M. MUDZIIRI SHUMBA, Department of Pure and Applied Mathematics, University of Johannesburg, Auckland Park, Johannesburg, 2006, South Africa e-mail: tmudziirishumba@uj.ac.za

M. C. PEDRAZA-AGUILERA, Instituto Universitario de Matemática Pura y Aplicada, Universitat Politècnica de València, 46022 Camino de Vera, València, Spain e-mail: mpedraza@mat.upv.es 